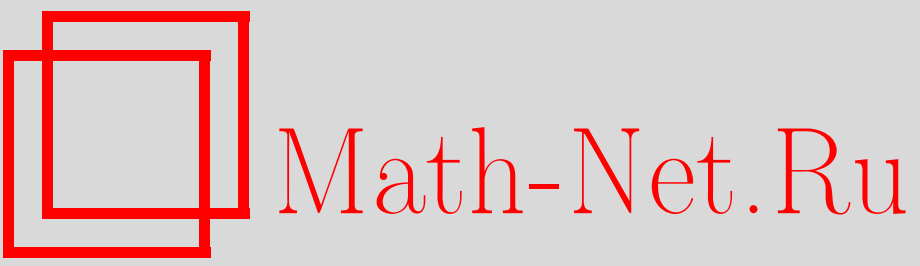

В. В. Подъяпольский, А. Л. Скубачевский, О полноте и базисности системы корневых функций сильно эллиптических функционально-дифференциальных операторов, УМH, 1996, том 51, выпуск 6, 219-220

DOI: https://doi.org/10.4213/rm1036

Использование Общероссийского математического портала Math-Net.Ru подразумевает, что вы прочитали и согласны с пользовательским соглашением

http://www.mathnet.ru/rus/agreement

Параметры загрузки:

IP: 54.162 .127 .20

26 апреля 2023 г., 14:01:27 


\title{
О ПОЛНОТЕ И БАЗИСНОСТИ СИСТЕМЫ КОРНЕВЫХ ФУНКЦИЙ СИЛЬНО ЭЛЛИПТИЧЕСКИХ ФУНКЦИОНАЛЬНО-ДИФФЕРЕНЦИАЛЬНЫХ ОПЕРАТОРОВ
}

\author{
В. В. ПОДъЯПОЛЬСКИЙ, А. Л. СКУБАЧЕВСКИЙ
}

Получены достаточные условия полноты и базисности по Абелю системы корневых функций сильно эллиптических (см. [1]) функционально-дифференциальных операторов.

1. Вначале сформулируем некоторые вспомогательные результаты об абстрактных $m$-секториальных операторах [2, гл. V, $\S 3$ и гл. VI, $\S 2]$. Пусть $H$ и $H_{1}$ - сепарабельные комплексные гильбертовы пространства со скалярными произведениями $(\cdot, \cdot)$ и $(\cdot, \cdot)_{1}$ и нормами $\|\cdot\|$ и $\|\cdot\|_{1}$, соответственно. Будем предполагать, что $H_{1}$ плотно в $H$ и оператор вложения компактный.

Пусть $a[u, v]$ - полуторалинейная форма в $H$ с областью определения $\mathscr{D}(a)=H_{1}$ такая, что

$$
|a[u, v]| \leqslant c_{0}\|u\|_{1}\|v\|_{1} \quad\left(u, v \in H_{1}\right), \quad c_{1}\|u\|_{1}^{2} \leqslant b[u, u] \quad\left(u \in H_{1}\right),
$$

где $c_{0}, c_{1}>0$ - постоянные, $b[u, v]$ - вещественная часть формы $a[u, v]$.

Из неравенств (1) следует, что форма $b$ - симметрическая, а форма $a$ - секториальная, т.е. числовая область значений формы $a$ лежит в секторе $|\arg \lambda| \leqslant \theta_{a}$, где $\theta_{a} \leqslant \operatorname{arctg}\left(c_{0} / c_{1}\right)<\pi / 2$. В силу первой теоремы о представлении [2, гл. VI, $\S 2]$ можно однозначно определить операторы $\mathscr{A}$ и $\mathscr{B}$ с плотными в $H$ областями определения, ассоциированные с формами $a$ и $b$, т.е.

$$
\begin{aligned}
& a[u, v]=(\mathscr{A} u, v) \quad\left(u \in \mathscr{D}(\mathscr{A}) \subset H_{1}, \quad v \in H_{1}\right), \\
& b[u, v]=(\mathscr{B} u, v) \quad\left(u \in \mathscr{D}(\mathscr{B}) \subset H_{1}, \quad v \in H_{1}\right),
\end{aligned}
$$

при этом оператор $\mathscr{A}-m$-секториален, а $\mathscr{B}$ - самосопряженньй, положительно определенный оператор. В силу сделанных предположений операторы $\mathscr{A}$ и $\mathscr{B}$ имеют компактные резольвенты в $H$. Следовательно, спектры $\sigma(\mathscr{A})$ и $\sigma(\mathscr{B})$ - дискретны.

Лемма 1. Пусть выполнены указанные выше предположсения, и пусть собственные значения $\lambda_{l}=\lambda_{l}(\mathscr{B})$ оператора $\mathscr{B}$, занумерованные в порядке неубывания и с учетом их кратности, удовлетворяют неравенству

$$
c_{2} l^{1 / p} \leqslant \lambda_{l}(\mathscr{B}) \leqslant c_{3} l^{1 / p} \quad\left(l \geqslant l_{0}\right),
$$

где $c_{2}, c_{3}>0$ - постоянные, $l_{0}>0$ - достаточно велико, $1 \leqslant p<\pi / 2 \theta_{a}$. Тогда система корневых функций оператора $\mathscr{A}$ полна в Н. Более того, в пространстве Н существует базис Абеля со скобками порядка $\gamma$, состоящий из әтих функций, для любого $\gamma$ такого, что $p<\gamma<\pi / 2 \theta_{a}$.

В силу теоремы 6.1 из [3, гл. $\mathrm{V}, \S 6]$ о полноте корневых функций компактного оператора и теоремы 3 из $[4, \S 5]$ о существовании базиса Абеля со скобками для доказательства леммы 1 достаточно установить оценку $s_{l}\left(\mathscr{A}^{-1}\right) \leqslant c_{4} l^{-1 / p}$ для $s$-чисел оператора $\mathscr{A}^{-1}$, где $c_{4}>0$ - постоянная. Вывод этой оценки аналогичен доказательству теоремы 1 из [5] и опирается на неравенство (2).

2. Пусть теперь $Q \subset \mathbb{R}^{n}$ - ограниченная область с границей $\partial Q \in C^{\infty}$. Введем ограниченные разностные операторы $R_{\alpha \beta}: L_{2}\left(\mathbb{R}^{n}\right) \rightarrow L_{2}\left(\mathbb{R}^{n}\right)$ и $R_{\alpha \beta Q}: L_{2}(Q) \rightarrow L_{2}(Q)$ по формулам

$$
R_{\alpha \beta} u(x)=\sum_{h \in \mathscr{M}} a_{\alpha \beta h}(x) u(x+h), \quad R_{\alpha \beta Q} v=P_{Q} R_{\alpha \beta} I_{Q} v .
$$

Здесь $\mathscr{M} \subset \mathbb{R}^{n}$ - конечное множество векторов с целочисленными координатами; $a_{\alpha \beta} \in C^{\infty}\left(\mathbb{R}^{n}\right)$ - комплекснозначные функция; $I_{Q}$ - оператор продолжения функций из $L_{2}(Q)$ нулем в $\mathbb{R}^{n} \backslash Q$; $P_{Q}$ - оператор сужения функций из $L_{2}\left(\mathbb{R}^{n}\right)$ на $Q$.

Работа выполнена при финансовой поддержке Российского фонда фундаментальных исследований (грант № 95-01-00247). 
Рассмотрим неограниченный дифференциально-разностный оператор $\mathscr{A}_{R}: L_{2}(Q) \rightarrow L_{2}(Q)$ с областью определения $\mathscr{D}\left(\mathscr{A}_{R}\right)=\left\{u \in \stackrel{\circ}{W} \underset{2}{m}(Q): \mathscr{A}_{R} u \in L_{2}(Q)\right\}$, действующий в пространстве обобщенных функций $\mathscr{D}^{\prime}(Q)$ по формуле

$$
\mathscr{A}_{R} u=\sum_{|\alpha|,|\beta| \leqslant m} \mathscr{D}^{\alpha} R_{\alpha \beta Q^{2}} \mathscr{D}^{\beta} u \quad\left(u \in \mathscr{D}\left(\mathscr{A}_{R}\right)\right),
$$

где $\stackrel{\circ}{W} \underset{2}{m}(Q)$ - замыкание множества финитных бесконечно дифференцируемых функций $\dot{C}^{\infty}(Q)$ в комплексном пространстве Соболева $W_{2}^{m}(Q), \mathscr{D}^{\alpha}=\mathscr{D}_{1}^{\alpha_{1}} \ldots \mathscr{D}_{n}^{\alpha_{n}}, \mathscr{D}_{j}=-i\left(\partial / \partial x_{j}\right)$.

ОПРЕДЕЛЕниЕ 1 . Будем называть оператор $\mathscr{A}_{R}$ сильно эллиптическим в $\bar{Q}$, если существуют постоянные $C_{1}>0$ и $C \geqslant 0$ такие, что

$$
\operatorname{Re}\left(\mathscr{A}_{R} u, u\right)_{L_{2}(Q)} \geqslant C_{1}\|u\|_{W_{2}^{m}(Q)}^{2}-C\|u\|_{L_{2}(Q)}^{2} \quad\left(u \in \dot{C}^{\infty}(Q)\right)
$$

В работе [1] получены необходимые и достаточные условия сильной эллиптичности.

Введем полуторалинейную форму $a_{R}[u, v]$ с областью определения $\mathscr{D}\left(a_{R}\right)=\stackrel{\circ}{W_{2}^{m}}(Q)$ :

$$
a_{R}[u, v]=\sum_{|\alpha|,|\beta| \leqslant m}\left(R_{\alpha \beta Q} \mathscr{D}^{\beta} u, \mathscr{D}^{\alpha} v\right)_{L_{2}(Q)}+C(u, v)_{L_{2}(Q)} .
$$

Если оператор $\mathscr{A}_{R}$ - сильно эллиптический в $\bar{Q}$, то форма $a_{R}$ - замкнута, а ее числовая область значений лежит в некотором секторе $|\arg \lambda| \leqslant \theta_{R}$, где $\theta_{R}<\pi / 2$.

Teорема 1. Пусть оператор $\mathscr{A}_{R}: L_{2}(Q) \rightarrow L_{2}(Q)-$ сильно әллиптический в $\bar{Q}, u$ пусть $n / m<\pi / \theta_{R}$. Тогда система корневых функций оператора $\mathscr{A}_{R}$ полна в $L_{2}(Q)$. Более того, в пространстве $L_{2}(Q)$ существует базис Абеля со скобками порядка $\gamma$, состоящий из этих функций, для любого $\gamma$ такого, что $n / 2 m<\gamma<\pi / 2 \theta_{R}$.

Для доказательства заметим, что оператор $\mathscr{A}=\mathscr{A}_{R}+C I-m$-секториальный оператор, ассоциированный с формой $a=a_{R}$. Чтобы применить лемму 1 , достаточно установить оценку

$$
c_{4} l^{2 m / n} \leqslant \lambda_{l}(\mathscr{B}) \leqslant c_{5} l^{2 m / n}
$$

где $c_{4}, c_{5}>0$ - постоянные, $\mathscr{B}$ - самосопряженный, положительно определенный оператор, ассоциированный с формой

$$
b_{R}[u, v]=\frac{1}{2} \sum_{|\alpha|,|\beta| \leqslant m}\left(\left(R_{\alpha \beta Q}+R_{\beta \alpha Q}^{*}\right)^{\beta} u, \mathscr{D}^{\alpha} v\right)_{L_{2}(Q)}+C(u, v)_{L_{2}(Q)},
$$

где $\mathscr{D}\left(b_{R}\right)=\stackrel{\circ}{W} \underset{2}{m}(Q)$. Оценку (3) легко получить из асимптотической формулы для собственных значений оператора $(-\Delta)^{m}$ в области $Q$ с условиями Дирихле на границе $\partial Q$ и минимаксных свойств собственных значений [6, гл. VI, п. 95].

Полученные результаты обобшаются на случай функционально-дифференциального оператора $\mathscr{A}_{R}$, содержащего растяжения и сжатия аргументов, рассмотренного в [7].

Авторы глубоко благодарны М. С. Аграновичу за обсуждение работы и ряд ценных советов.

\section{СПИСОК ЛИТЕРАТУРЫ}

[1] Skubachevskii A.L. // J. Differential Equations. 1986. V. 63. №3. P. 332-361. [2] Като Т. Теория возмущений линейных операторов. М.: Мир, 1972. [3] Гохберг И. Ц., Крейн М. Г. Введение в теорию линейных несамосопряженных операторов. М.: Наука, 1965. [4] Лидский В. Б. // Труды ММО. 1962. Т. 11. С. 3-35. [5] Agranovich M. S. // Russian J. Math. Physics. 1994. V. 2. № 2. Р. 139-148. [6] Рисс Ф., Секефальви-Надь Б. Лекции по функциональному анализу. М.: Мир, 1979. [7] Россовский Л.Е. // Матем. заметки. 1996. T. 56. №1. C. 103-113. 